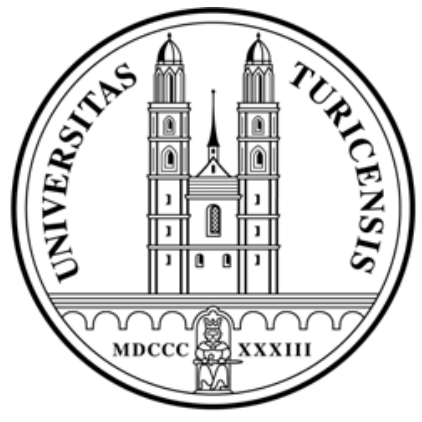

Institute for Empirical Research in Economics

University of Zurich

Working Paper Series

ISSN 1424-0459

Working Paper No. 409

\title{
Optimal Design and $\rho$-Concavity
}

Christian Ewerhart

May 2011 


\title{
Optimal design and $\rho$-concavity*
}

\author{
Christian Ewerhart**
}

*) For useful comments, I am grateful to participants of the 2009 Far East and South Asia Meeting of the Econometric Society in Tokyo.

**) University of Zurich; postal address: Chair for Information Economics and Contract Theory, Winterthurerstrasse 30, CH-8006 Zurich, Switzerland; e-mail: christian.ewerhart@econ.uzh.ch, phone: 41-44-6343733; fax: 41-446344978. 


\title{
Optimal design and $\rho$-concavity
}

\begin{abstract}
Some of the most beautiful results in mechanism design depend crucially on Myerson's (1981) regularity condition. E.g., the second-price auction with reserve price is revenue maximizing only if the type distribution is regular. This paper offers two main results. First, an interpretation of regularity is developed in terms of being the next to fail. Second, using expanded concepts of concavity, a tight sufficient condition on the density function is formulated. New examples of parameterized distributions are shown to be regular. Applications include standard design problems, optimal reserve prices, the analysis of bidding data, and multidimensional types.
\end{abstract}

Keywords and Phrases. Virtual valuation, Regularity, Generalized concavity, Prékopa-Borell Theorem, Mechanism design.

JEL-Codes. D82 - Asymmetric and Private Information; D44 - Auctions; D86 - Economics of Contract: Theory; C16 - Specific Distributions. 


\section{Introduction}

Some of the best-known results in the theory of mechanism design require that the underlying type distribution be regular. For example, the secondprice auction with reserve price is revenue maximizing only under the condition of regularity. ${ }^{1}$ It is therefore of some interest to clarify the meaning and scope of the assumption. Formally, regularity says that the virtual valuation,

$$
J_{f}(x)=x-\frac{1-F(x)}{f(x)},
$$

is strictly increasing in the type $x$, where $f$ and $F$, respectively, denote the density and distribution function of the type distribution. ${ }^{2}$

A simple way to ensure regularity is to impose that the hazard rate of the type distribution,

$$
\lambda_{f}(x)=\frac{f(x)}{1-F(x)},
$$

is monotone increasing. This approach has been found useful mainly for two reasons. First, the hazard rate allows an immediate interpretation as a conditional likelihood of failure. ${ }^{3}$ Second, distributions with log-concave densities are known to possess a monotone hazard rate. ${ }^{4}$

However, the hazard rate condition will often be more restrictive than necessary. For example, the log-normal distribution does not possess a monotone

\footnotetext{
${ }^{1}$ Cf. Myerson [15]. When the type distribution is not regular, the optimal mechanism will typically entail conditional minimum bids, see Maskin and Riley [13].

${ }^{2}$ Equivalently, the marginal revenue of a monopolist facing inverse demand $p=F^{-1}(1-$ $q)$ is strictly declining in output. See Bulow and Roberts [5].

${ }^{3}$ Indeed, if $F(x)$ is the probability that a machine will fail before time $x$, then the hazard rate is the instantaneous probability of failure, given that the machine has not failed before time $x$. See, e.g., Barlow and Proschan [3].

${ }^{4}$ See, e.g., An [1]. This result can be used to identify many parameterized examples of regular type distributions. Specifically, as Bagnoli and Bergström [2] show, regularity holds for the uniform, normal, exponential, logistic, extreme value, Laplace, Maxwell, and Rayleigh distributions. With restrictions to parameters, this list extends to power, Weibull, Gamma, Chi-Squared, Chi, and beta distributions.
} 
hazard rate (cf. Figure 1), but will still be regular unless heavily skewed. ${ }^{5}$ Thus, imposing the hazard rate condition not only curtails the strength of theoretical results, but also restricts unnecessarily the range of distributional assumptions in empirical work.

The present paper offers two main results. The first is a statistical interpretation of regularity. More specifically, we characterize the assumption in terms of the probability of being the next to fail, conditional on not having failed before. The second main result is a sufficient criterion on the density that is substantially tighter than log-concavity. With the help of that criterion, new examples of parameterized distributions can be shown to be regular.

The rest of the paper is organized as follows. Section 2 reviews mathematical prerequisites. An interpretation of regularity is developed in Section 3. In Section 4, we prove a general characterization of distributions that possess monotone virtual valuations. A tight criterion on the density is derived and discussed in Section 5. Section 6 outlines some applications. An Appendix deals with examples of parameterized distributions.

\section{Mathematical tools}

This section reviews some mathematical concepts and results that will be used in the analysis.

\subsection{Generalized concavity}

A function $g \geq 0$ on $\mathbb{R}^{N}$ is called $\rho$-concave, for $\rho \neq 0$, if the set $X_{g}=$ $\left\{x \in \mathbb{R}^{N}: g(x)>0\right\}$ is convex, and $g^{\rho} / \rho$ is concave on $X_{g}$. For $\rho=0$,

\footnotetext{
${ }^{5}$ In fact, our results imply that the log-normal distribution is regular provided that skewness is smaller than $\left(e^{2}+2\right) \sqrt{e^{2}-1} \approx 23.73$.
} 
the definition is extended by the requirement that $g$ must be log-concave. ${ }^{6}$ For a twice differentiable $g>0$, the condition of $\rho$-concavity is equivalent to $g(x) g^{\prime \prime}(x) \leq(1-\rho) g^{\prime}(x)^{2}$.

One can show that higher values of $\rho$ correspond to more stringent variants of concavity. In particular, log-concavity is more stringent than $\rho$ concavity for any $\rho<0$. We call a function $g$ strongly $\rho$-concave if $g$ is $\rho^{\prime}$-concave for some $\rho^{\prime}>\rho$.

Among alternative notions of concavity, the definition above is highlighted by the fact that concavity properties are passed on from densities to integrals.

Theorem 2.1. (Prékopa-Borell) Let some $\rho$-concave density $g(x)$ on $\mathbb{R}^{N}$ be given, where $\rho>-\frac{1}{N}$. Then

$$
G(z)=\int_{\left\{x \in \mathbb{R}^{N}: x_{N} \leq z\right\}} g\left(x_{1}, \ldots, x_{N}\right) d x_{1} \ldots d x_{N}
$$

is $\widehat{\rho}$-concave with $\widehat{\rho}=\frac{\rho}{1+N \rho}$.

For a helpful discussion of this result, see Caplin and Nalebuff [6]. Obviously, the simplest version of the Prékopa-Borell Theorem says that if $g\left(x_{1}\right) \geq 0$ is $\rho$-concave for some $\rho>-1$, then $G(z)=\int_{-\infty}^{z} g\left(x_{1}\right) d x_{1}$ is $\widehat{\rho}$-concave with $\widehat{\rho}=\frac{\rho}{1+\rho}$.

\subsection{Minimal conditions for monotonicity}

A smooth function is monotone increasing provided that its first derivative is never negative. Here is a generalization to functions that are not differentiable. For a function $g$, write $\bar{g}^{+}(x)=\lim \sup _{\varepsilon \rightarrow 0+} \frac{1}{\varepsilon}(g(x+\varepsilon)-g(x))$ for the right-hand upper Dini derivative at $x$.

\footnotetext{
${ }^{6}$ This is the definition used in the economics literature since Caplin and Nalebuff $[6,7]$. E. Dierker [8] is an early application of generalized concavity in the economics literature.
} 
Theorem 2.2. Assume that

$$
\lim \sup _{\varepsilon \rightarrow 0+} g(x-\varepsilon) \leq g(x) \leq \lim \sup _{\varepsilon \rightarrow 0+} g(x+\varepsilon)
$$

at any $x$, that $\bar{g}^{+}(x) \geq 0$ a.e., and that $\bar{g}^{+}(x)>-\infty$ except possibly at a countable set. Then $g$ is monotone increasing.

This result is Theorem 7.3 in Saks $\left[18\right.$, Ch. VI]. ${ }^{7}$ Note that (4) holds if $g$ is right-continuous and upper semi-continuous.

\section{An interpretation of regularity}

The analysis starts from an observation due to McAfee and McMillan [12] saying that in the smooth case, regularity is equivalent to the strict convexity of $1 /(1-F)$. For a direct proof of this fact, assume that $F$ is twice differentiable. Then

$$
\frac{\partial J_{f}(x)}{\partial x}=\frac{\partial}{\partial x}\left(x-\frac{1-F(x)}{f(x)}\right)=1+\frac{f(x)^{2}+(1-F(x)) f^{\prime}(x)}{f(x)^{2}} .
$$

However,

$$
\frac{\partial^{2}}{\partial x^{2}} \frac{1}{1-F(x)}=\frac{\partial}{\partial x} \frac{f(x)}{(1-F(x))^{2}}=\frac{(1-F(x)) f^{\prime}(x)+2 f(x)^{2}}{(1-F(x))^{3}},
$$

i.e., the respective signs of $J_{f}^{\prime}$ and $(1 /(1-F))^{\prime \prime}$ coincide.

It apparently went unnoticed that this characterization implies the following statistical interpretation of regularity. There is a large number $M$ of machines which fail one after another at rate $f$. Pick one machine from the population, and assume it had been functional up to time $x$. By the law of large numbers, there are about $M(1-F(x))$ machines left. Hence, the likelihood for the chosen machine to be the next to fail is $l(x) \approx 1 / M(1-F(x))$.

\footnotetext{
${ }^{7}$ See also the discussion following the theorem.
} 
Call the rate at which the likelihood $l(x)$ grows over time the "zoom rate." Then regularity requires the zoom rate to be strictly increasing over time. ${ }^{8}$

\section{A generalization}

So far, we assumed that the density function is differentiable. However, this may be restrictive, e.g., when the distribution is a mixture or the result of endogenous decisions. To incorporate such possibilities, we will now replace smoothness by a somewhat weaker assumption. Obviously, this section can be skipped by readers interested only in the main results.

Consider a density $f \geq 0$ on some interval $X$. Clearly, there is no loss in assuming that $f$ is strictly positive everywhere in the interior of $X$. Indeed, if $f(x)=0$ at some interior point, then $J_{f}(x)=-\infty$, and $J_{f}$ cannot be increasing. We will say that $f$ satisfies the Cantor-Lebesgue condition (CL) if $f$ is right-continuous in the interior of $X$, upper semi-continuous, and satisfies $\bar{f}^{+}>-\infty$ except possibly at a countable set. This condition is obviously very weak. For example, it is satisfied for right-continuous, piecewise differentiable densities that do not possess downward jumps. ${ }^{9}$

\footnotetext{
${ }^{8}$ Here is a somewhat more rigorous argument. Let $m \geq 1$ denote the number of machines that are still working at time $x$. Then

$$
\begin{aligned}
l(x) & =\sum_{m=1}^{M} \frac{1}{m}\left(\begin{array}{c}
M-1 \\
m-1
\end{array}\right)(1-F(x))^{m-1} F(x)^{M-m} \\
& =\frac{1}{M(1-F(x))} \sum_{m=1}^{M}\left(\begin{array}{c}
M \\
m
\end{array}\right)(1-F(x))^{m} F(x)^{M-m} \\
& =\frac{1-F(x)^{M}}{M(1-F(x))} .
\end{aligned}
$$

Therefore, for $x$ kept constant, the product $M(1-F(x)) l(x)$ converges to unity as $M \rightarrow \infty$. Further, one can check that $\partial l / \partial x \approx f(x) / M(1-F(x))^{2}$. This follows from differentiating the precise expression for $l(x)$ derived above.

${ }^{9}$ Monteiro and Svaiter [14] study optimal design for arbitrary distributions. For example, the support of the distribution may have gaps, and there may be mass points.
} 
The following auxiliary result can be seen as a generalization of the smooth characterization of regularity. Note, however, that it concerns weakly increasing virtual valuations.

Lemma 4.1. Let $f>0$ be a density on some interval $X$, and assume that condition $(C L)$ holds. Then, $J_{f}(x)$ is nondecreasing if and only if $1 /(1-$ $F(x))$ is convex.

Proof. The right-hand upper Dini derivative of $J_{f}(x)=x-(1-F(x)) / f(x)$ is given by

$$
\begin{aligned}
\bar{J}_{f}^{+}(x) & =1+\lim \sup _{\varepsilon \rightarrow 0+} \frac{1}{\varepsilon}\left\{-\frac{1-F(x+\varepsilon)}{f(x+\varepsilon)}+\frac{1-F(x)}{f(x)}\right\} \\
& =1+\lim \sup _{\varepsilon \rightarrow 0+} \frac{1}{f(x+\varepsilon)}\left\{\frac{F(x+\varepsilon)-F(x)}{\varepsilon}+\right. \\
& \left.+\frac{1-F(x)}{f(x)} \frac{f(x+\varepsilon)-f(x)}{\varepsilon}\right\} .
\end{aligned}
$$

Clearly, the derivative of $F$ is a.e. well-defined with $F^{\prime}=f$. Hence, noting that $f$ is right-continuous,

$$
\bar{J}_{f}^{+}(x)=2+\frac{(1-F(x)) \bar{f}^{+}(x)}{f(x)^{2}}
$$

a.e. in $X$. Let $\vartheta_{f}(x)=f(x) /(1-F(x))^{2}$. Then the right-hand upper Dini derivative of $\vartheta_{f}(x)$ is

$$
\begin{aligned}
\bar{\vartheta}_{f}^{+}(x) & =\lim _{\varepsilon \rightarrow 0+} \frac{1}{\varepsilon}\left\{\frac{f(x+\varepsilon)}{(1-F(x+\varepsilon))^{2}}-\frac{f(x)}{(1-F(x))^{2}}\right\} \\
& =\frac{1}{(1-F(x))^{2}} \lim \sup _{\varepsilon \rightarrow 0+}\left\{\frac{f(x+\varepsilon)-f(x)}{\varepsilon}+\right. \\
& \left.+\frac{f(x)}{(1-F(x))^{2}} \frac{(1-F(x))^{2}-(1-F(x+\varepsilon))^{2}}{\varepsilon}\right\} .
\end{aligned}
$$

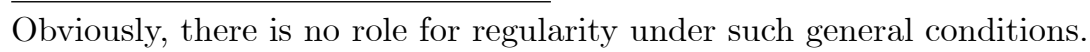


Hence,

$$
\bar{\vartheta}_{f}^{+}(x)=\frac{\bar{f}^{+}(x)(1-F(x))+2 f(x)^{2}}{(1-F(x))^{3}}
$$

a.e. in $X$. Comparing (9) and (12) shows that $\bar{J}_{f}^{+}(x)$ and $\bar{\vartheta}_{f}^{+}(x)$ share the same sign a.e. in $X$.

"Only if." Assume that $J_{f}$ is monotone. Then $\bar{J}_{f}^{+} \geq 0$ on $X$, and therefore, $\bar{\vartheta}_{f}^{+}(x) \geq 0$ a.e. in $X$. An inspection of (11) shows that $\vartheta_{f}$ satisfies condition (CL). Hence, by Theorem 2.2, $\vartheta_{f}$ is nondecreasing. Thus, the integral $1 /(1-F(x))$ is convex.

"If." Conversely, assume that $1 /(1-F(x))$ is convex. Then the left derivative of $1 /(1-F(x))$ is well-defined in the interior of $X$ and monotone. But a.e. in $X$, the left derivative of $1 /(1-F(x))$ is given by $\vartheta_{f}$. Thus, $\bar{\vartheta}_{f}^{+}(x) \geq 0$ a.e. in $X$. As shown above, this implies $\bar{J}_{f}^{+}(x) \geq 0$ a.e. in $X$. One can check using (8) that $J_{f}$ satisfies condition (CL). Therefore, by another application of Theorem 2.2, $J_{f}$ is monotone increasing.

\section{A condition on the density}

In this section, we apply the Prékopa-Borell theorem to derive a tight criterion for regularity on the underlying density function. To deal with strict monotonicity, and to allow for modifications of the regularity assumption, we will write

$$
J_{f}(x, a, b)=a x-\frac{b-F(x)}{f(x)},
$$

where $a, b \in \mathbb{R}$. Obviously, $J_{f}(x, 1,1) \equiv J_{f}(x)$.

Theorem 5.1. Let $f>0$ be a density on some interval $X$, and $a>-1$. Then $J_{f}(x, a, b)$ is weakly increasing in $x$ [strictly increasing in $x$ ] for any 
$b \in[0,1]$ if $f$ is $\rho$-concave [strongly $\rho$-concave] for $\rho=-\frac{a}{1+a}$. In particular, $J_{f}(x)$ is strictly increasing if $f$ is strongly $\left(-\frac{1}{2}\right)$-concave.

Proof. Assume that $f$ is $\left(-\frac{a}{1+a}\right)$-concave for some $a>-1$. Consider the mirror image density $g(y)=f(-y)$. Obviously, also $g$ is $\left(-\frac{a}{1+a}\right)$-concave. By Theorem 2.1, the integral $G(y)=1-F(-y)$ is $(-a)$-concave, and so is $1-$ $F(x)$. Since $f$ must be continuous in $X$, with finite right derivative, condition (CL) holds. Therefore, in straightforward extension of Lemma 4.1, $J_{f}(x, a, 1)$ is nondecreasing. Similarly, $J_{f}(x, a, 0)$ is nondecreasing since $F(x)=1-$ $G(-x)$ is $(-a)$-concave. The unbracketed part of the theorem follows now from noting that $J_{f}(x, a, b)$ is linear in $b$. If $f$ is even strongly $\left(-\frac{a}{1+a}\right)$-concave for some $a>-1$ then, by the first part of the proof, $J_{f}\left(x, a^{\prime}, b\right)$ is weakly increasing in $x$ for some $a^{\prime} \in(-1, a)$. Hence, $J_{f}(x, a, b)=J_{f}\left(x, a^{\prime}, b\right)+(a-$ $\left.a^{\prime}\right) x$ is strictly increasing in $x$.

Thus, strong $\left(-\frac{1}{2}\right)$-concavity is sufficient for regularity. Since any log-concave density is strongly $\left(-\frac{1}{2}\right)$-concave, Theorem 5.1 clearly implies the conventional log-concavity criterion. ${ }^{10}$

To test the power of the square root criterion, we applied it to various examples of parameterized distributions that do not allow a log-concave density. ${ }^{11}$ As Table I shows, regular design is feasible in particular for the lognormal, Pareto, log-logistic, Student, Cauchy, F, beta prime, mirror-image Pareto, inverse gamma, inverse chi-squared, and Pearson distributions, where

\footnotetext{
${ }^{10}$ Theorem 5.1 can also be applied if the density function has finitely many convex kinks and jump discontinuities. In such cases, one requires strong $\left(-\frac{1}{2}\right)$-concavity of $f$ in each smooth segment, and strong $(-1)$-concavity of $F$ just left of critical points. For a proof, one constructs a strongly $\left(-\frac{1}{2}\right)$-concave extension of the density right of the critical point. The details are omitted.

${ }^{11}$ See the Appendix for details.
} 
constraints on the parameters typically ensure that these distributions are not too much skewed. Conversely, Table II lists various distributions that lack a strongly $\left(-\frac{1}{2}\right)$-concave density function. Most of those are not regular (i.e., exhibit either $J_{f}^{\prime} \nsupseteq 0, J_{f}^{\prime}<0$, or mixed results), which illustrates the tightness of the criterion. ${ }^{12}$

\section{Applications}

This section illustrates the use of Theorem 5.1 in specific settings.

6.1. Standard mechanism design. Here are three immediate applications. First, the revenue-maximizing mechanism in Myerson [15] is a second-price auction with reserve price if the density of types is strongly $\left(-\frac{1}{2}\right)$-concave. Second, an optimal trading mechanism exists in Myerson and Satterthwaite [16] if the buyer's virtual valuation and the seller's virtual cost $\bar{J}_{f}(x)=$ $x+\frac{F(\theta)}{f(\theta)}$ are both increasing. Again, a strongly $\left(-\frac{1}{2}\right)$-concave density suffices. Finally, optimal regulation discriminates between cost types in Baron and Myerson [4] if $\theta+(1-\alpha) \frac{F(\theta)}{f(\theta)}$ is increasing in $\theta$, where $\alpha$ is the policy weight of monopoly. It suffices that $f$ is $\rho$-concave for $\rho>-\frac{1}{2-\alpha}$.

6.2. Optimal reserve prices. Riley and Samuelson [17] show that the optimal reserve price in a broad class of auctions can be found by setting the virtual valuation equal to the seller's reservation value. The resulting equation has a unique root if the type distribution is strongly $\left(-\frac{1}{2}\right)$-concave.

6.3. Empirical analysis of bidding data. Guerre et al. [10] prove that bids in a first-price auction can be rationalized as a Bayesian equilibrium under

\footnotetext{
${ }^{12}$ The square root criterion is indeed the tightest condition possible in terms of generalized concavity. E.g., for $\rho<-\frac{1}{2}$, take $f(x)=\beta x^{-\beta-1}$ on $X=[1, \infty)$ with $\beta=-\frac{\rho+1}{\rho}$. Note that $f(x)$ is $\rho$-concave. But $J_{f}(x)=\left(1-\frac{1}{\beta}\right) x$ is strictly decreasing since $\beta<1$.
} 
the independent private value paradigm if and only if $x+\frac{1}{I} \frac{G(x)}{g(x)}=\frac{J_{g}(x, I, 0)}{I}$ increases in $x$, where $I$ is the number of bidders, while $g$ and $G$, respectively, denote density and distribution of bids. It suffices that $g$ is strongly $\rho$-concave with $\rho=-\frac{I}{1+I}$.

6.4. Multidimensional types with externalities. An object is sold to one of $N$ buyers. With externalities, buyer $i$ 's type is a vector $\left(s_{i}^{i}, s_{-i}^{i}\right)$ whose entries specify the respective payoff to the buyer in case some buyer obtains the good. The distribution of buyer $i$ 's type follows some density $f_{i}\left(s_{i}^{i}, s_{-i}^{i}\right)$. Jehiel et al. [11] show that the revenue-maximizing standard anonymous mechanism that always transfers the object is a second-price auction with entry fee if a modified regularity condition holds. Specifically, for

$$
g(z)=\int_{\mathbb{R}^{N-1}} f_{i}\left(z+\frac{1}{N-1} \sum_{j \neq i} s_{j}^{i}, s_{-i}^{i}\right) d s_{-i}^{i},
$$

the virtual valuation $J_{g}$ needs to be increasing. By Theorem 5.1, it suffices that $g$ is strongly $\left(-\frac{1}{2}\right)$-concave. But the change of variables in the argument of $f_{i}$ effective in (14) is an affine transformation of $\mathbb{R}^{N}$, which leaves generalized concavity unaffected. Therefore, using Theorem 2.1, the second-price auction with entry fee is optimal if all $f_{i}$ are strongly $\left(-\frac{1}{N+1}\right)$-concave.

\section{Appendix. Parameterized distributions}

This appendix outlines the derivations underlying Tables I and II. The main tool is the following smooth criterion for $\rho$-concavity.

Lemma A.1. Let $f>0$ be twice continuously differentiable on $X$, with a discrete set $X_{1}$ over which $f^{\prime}(x)=0$. Then, for finite $\rho$, the function $f$ is $\rho$-concave if and only if $r_{f}(x) \equiv-\frac{\partial^{2}}{\partial x^{2}} \ln f(x) /\left(\frac{\partial}{\partial x} \ln f(x)\right)^{2} \geq \rho$ for all 
$x \in X \backslash X_{1}$.

Proof. A straightforward calculation shows that $r_{f}(x)=1-f(x) f^{\prime \prime}(x) / f^{\prime}(x)^{2}$. Hence, $r_{f}(x) \geq \rho$ if and only if $f(x) f^{\prime \prime}(x) \leq(1-\rho) f^{\prime}(x)^{2}$, provided $f^{\prime}(x) \neq 0$. By continuity, this proves the assertion.

Lemma A.1 reduces the determination of the global concavity parameter $\rho$ to the solution of a straightforward minimization problem. For convenience, explicit expressions of $r_{f}(x)$ are documented in Table I.

The following criterion is useful for identifying distributions for which $J_{f}(x)$ is not increasing.

Lemma A.2. Let $f>0$ be differentiable on $X$. Assume that $f^{\prime}(x)$ is negative and bounded away from zero in a neighborhood of the lower boundary of the support interval. Then $J_{f}(x)$ is not increasing.

Proof. Clearly $J_{f}^{\prime}(x)=2+f^{\prime}(x)(1-F(x)) / f(x)^{2}$. But $f(x)$ vanishes at the lower boundary of $X$.

We now use the results obtained so far to discuss selected examples of parameterized distributions. Unless noted otherwise, all parameters are strictly positive.

Log-normal distribution. The square root criterion implies that $J_{f}$ is increasing for $\sigma_{L}^{2} \leq 2$. Since $F$ is log-concave, $\bar{J}_{f}$ is increasing.

$F$ distribution. The density is $\left(-\frac{2}{2+m_{2}}\right)$-concave if $m_{1} \geq 2$. Thus, $J_{f}$ is monotone increasing for $m_{1}, m_{2} \geq 2$. For $m_{1}<2$, Lemma A.2 applies. Since $F$ is log-concave (cf. Finner and Roters $[9]), \bar{J}_{f}$ is increasing.

Gamma and Chi distributions. Only the case $c<1$ is interesting. By Lemma A.2, $J_{f}$ is not increasing. The density is decreasing, hence $\bar{J}_{f}$ is 
increasing.

Log-logistic. Since $1 /(1-F(x))=1+x^{\beta}$, it follows that $J_{f}$ is increasing if and only if $\beta>1$. Similarly, $1 / F(x)=1+x^{-\beta}$ implies that $\bar{J}_{f}$ is increasing.

Inverse gamma distribution. By the square root criterion, $J_{f}$ is monotone increasing for $\alpha \geq 1$. The density is log-concave for $x \leq x^{M}$, and decreasing for $x \geq x^{M}$. Hence, $\bar{J}_{f}$ is increasing.

Beta distribution. Only the cases with $\nu<1$ or $\omega<1$ are interesting. If $\nu<1$, Lemma A.2 applies. If $\nu>1>\omega$, then $f$ is increasing, hence so is $J_{f}$. The analysis for $\bar{J}_{f}$ is analogous.

Pearson distributions. The density solves the differential equation $f^{\prime}(x)=$ $f(x)\left(x-x^{M}\right) / \chi(x)$ for $x^{M} \in \mathbb{R}$ and $\chi(x)=b_{0}+b_{1} x+b_{2} x^{2}$, where $b_{0}, b_{1}, b_{2} \in \mathbb{R}$. We focus on distributions with unbounded support and such that $\chi\left(x^{M}\right)<0$. Then $f$ is $b_{2}$-concave. Both $J_{f}$ and $\bar{J}_{f}$ are increasing provided that $b_{2} \geq-\frac{1}{2}$. The analysis of the Pareto, mirror image Pareto, power, Student, and Weibull distributions is straightforward. The remaining distributions can be seen as either transforms or special cases of examples already discussed. 


\section{References}

[1] M.Y. An, Logconcavity versus logconvexity: a complete characterization, Journal of Economic Theory 80 (1998), 350-369.

[2] M. Bagnoli, T. Bergström, Log-concave probability and its applications, Economic Theory 26 (2005), 445-469.

[3] R.E. Barlow, F. Proschan, Statistical Theory of Reliability and Life Testing: Probability Models, Holt, Rinehart and Winston, New York, 1975 .

[4] D.P. Baron, R.B. Myerson, Regulating a monopolist with unknown costs, Econometrica 50 (1982), 911-930.

[5] J. Bulow, J. Roberts, The simple economics of optimal auctions, Journal of Political Economy 97 (1989), 1060-1090.

[6] A. Caplin, B. Nalebuff, Aggregation and social choice: a mean voter proposition, Econometrica 59 (1991), 1-23.

[7] A. Caplin, B. Nalebuff, Aggregation and imperfect competition: on the existence of equilibrium, Econometrica 59 (1991), 25-59.

[8] E. Dierker, Competition for customers, in: W.A. Barnett, B. Cornet, C. d'Aspremont, J. Gabszewicz, and A. Mas-Colell (Eds.), Equilibrium theory and applications: proceedings of the sixth International Symposium in Economic Theory and Econometrics. Cambridge University Press, Cambridge, 1991, pp. 383-402.

[9] H. Finner, M. Roters, Log-concavity and inequalities for chi-square, F and beta distributions with applications in multiple comparisons, Statistica Sinica 7 (1997), 771-787.

[10] E. Guerre, I. Perrigne, Q. Vuong, Optimal nonparametric estimation of first-price auctions, Econometrica 68 (2000), 525-574. 
[11] P. Jehiel, B. Moldovanu, E. Stacchetti, Multidimensional mechanism design for auctions with externalities, Journal of Economic Theory 85 (1999), 258-293.

[12] McAfee, P., McMillan, J., 1987, Auctions and bidding, Journal of Economic Literature 25, 699-738.

[13] E. Maskin, J. Riley, Optimal multi-unit auctions, in: F. Hahn (Ed.), The Economics of Missing Markets, Information, and Games. Clarendon Press, Oxford, 1989, pp. 312-335.

[14] P.K. Monteiro, B.F. Svaiter, Optimal auction with a general distribution: Virtual valuation without densities, Journal of Mathematical Economics 46 (2010), 21-31.

[15] R. Myerson, Optimal auction design, Mathematics of Operations Research 6 (1981), 58-73.

[16] R. Myerson, M. Satterthwaite, Efficient mechanisms for bilateral trading, Journal of Economic Theory 29 (1983), 265-281.

[17] J. Riley, W. Samuelson, Optimal auctions, American Economic Review 71 (1981), 381-392.

[18] S. Saks, Theory of the Integral, Hafner Publishing, New York, 1937. 
Figure 1. Regularity of the log-normal distribution. ${ }^{\dagger}$

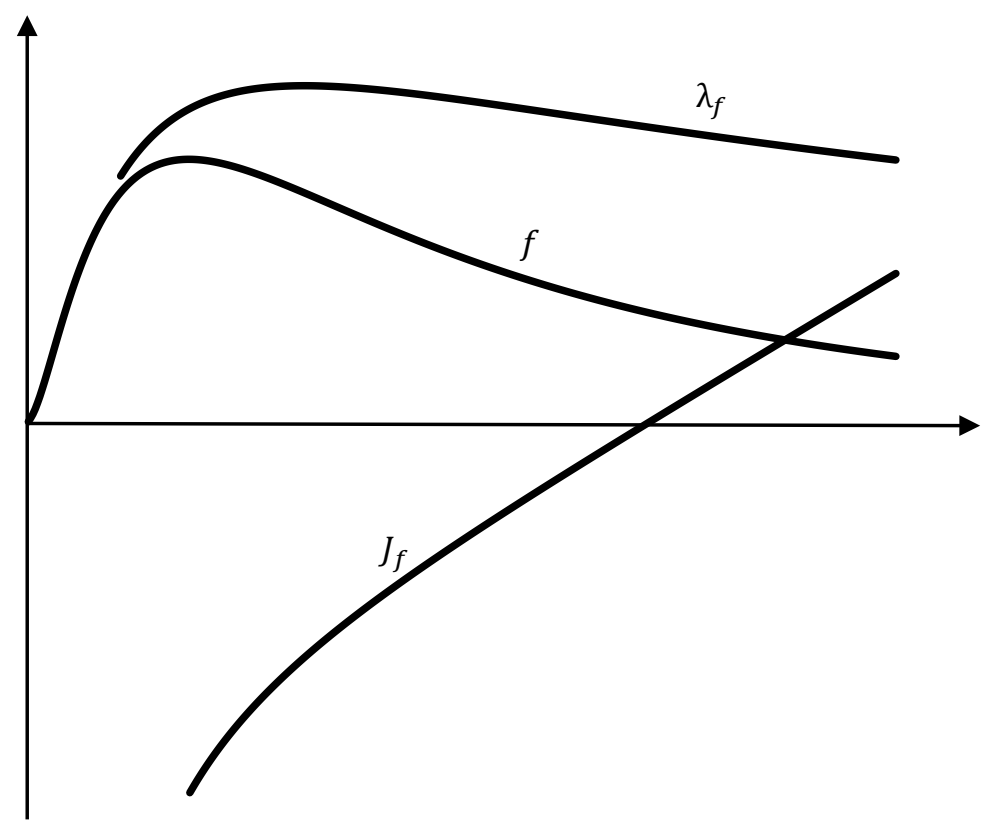

† The figure shows density, hazard rate, and virtual valuation of a random variable whose logarithm follows a standard normal distribution. 
Table I. Distributions with strongly $\left(-\frac{1}{2}\right)$-concave density function. ${ }^{\dagger}$

\begin{tabular}{|c|c|c|c|c|c|c|}
\hline Name of distribution & $\begin{array}{c}\text { Interval } \\
X\end{array}$ & $\begin{array}{l}\text { P.d.f. } \\
f(x)\end{array}$ & $\begin{array}{l}\text { C.d.f. } \\
F(x)\end{array}$ & $\begin{array}{c}\text { Mode } \\
x^{M}\end{array}$ & $\begin{array}{l}\text { Local concavity } \\
\qquad r_{f}(x)\end{array}$ & $\begin{array}{c}\text { Global } \\
\text { concavity } \\
\rho \\
\end{array}$ \\
\hline $\begin{array}{l}\text { Any with log-concave } \\
\text { density }\end{array}$ & \multicolumn{4}{|c|}{ - see Bagnoli and Bergström (2006, Table 1) - } & $\geq 0$ & $\geq 0$ \\
\hline Pareto $(\beta>1)$ & {$[1 ; \infty)$} & $\beta x^{-\beta-1}$ & $1-x^{-\beta}$ & 1 & $-\frac{1}{\beta+1}$ & $-\frac{1}{\beta+1}$ \\
\hline Log-normal $\left(\sigma_{L}^{2}<2\right)$ & {$[0 ; \infty)$} & $\propto \frac{1}{x} \exp \left(-\frac{\left(\ln x-\mu_{L}\right)^{2}}{2 \sigma_{L}^{2}}\right)$ & $*$ & $\exp \left(\mu_{\mathrm{L}}-\sigma_{\mathrm{L}}^{2}\right)$ & $\sigma_{L}^{2} \frac{1-\ln \left(x / x^{M}\right)}{\ln \left(x / x^{M}\right)^{2}}$ & $-\frac{\sigma_{L}^{2}}{4}$ \\
\hline Student's $t(n>1)$ & $\mathbb{R}$ & $\propto\left(1+\frac{x^{2}}{n}\right)^{-\frac{n+1}{2}}$ & $*$ & 0 & $\frac{n-x^{2}}{(n+1) x^{2}}$ & $-\frac{1}{n+1}$ \\
\hline Cauchy & $\mathbb{R}$ & $\frac{1}{\pi\left(1+x^{2}\right)}$ & $\frac{1}{2}+\frac{\arctan x}{\pi}$ & 0 & $\frac{1-x^{2}}{2 x^{2}}$ & $-\frac{1}{2}$ \\
\hline $\begin{array}{l}\text { F distribution } \\
\left(m_{1}, m_{2}>2\right)\end{array}$ & {$[0 ; \infty)$} & $\propto \frac{x^{\frac{m_{1}}{2}-1}}{\left(m_{1} x+m_{2}\right)^{\frac{m_{1}+m_{2}}{2}}}$ & $*$ & $\frac{\left(m_{1}-1\right) m_{2}}{m_{1}\left(m_{2}+2\right)}$ & $\frac{2}{m_{2}+2}\left(\frac{x^{M}\left(x^{M}+m_{2} / m_{1}\right)}{\left(x-x^{M}\right)^{2}}-1\right)$ & $-\frac{2}{m_{2}+2}$ \\
\hline $\begin{array}{l}\text { Mirror-image of } \\
\text { Pareto }(\beta>1)\end{array}$ & $(-\infty ;-1]$ & $\beta(-x)^{-\beta-1}$ & $(-x)^{-\beta}$ & -1 & $-\frac{1}{\beta+1}$ & $-\frac{1}{\beta+1}$ \\
\hline Log-logistic $(\beta>1)$ & {$[0 ; \infty)$} & $\frac{\beta x^{\beta-1}}{\left(1+x^{\beta}\right)^{2}}$ & $\frac{1}{1+x^{-\beta}}$ & $\left(\frac{\beta-1}{\beta+1}\right)^{1 / \beta}$ & $\frac{\beta^{2}-(1+\beta)\left(x^{\beta}-\beta+1\right)^{2}}{\left(x^{\beta}(\beta+1)-\beta+1\right)^{2}}$ & $-\frac{1}{\beta+1}$ \\
\hline $\begin{array}{l}\text { Inverse gamma } \\
(\alpha>1)\end{array}$ & {$[0 ; \infty)$} & $\frac{\exp (-1 / x)}{\Gamma(\alpha) x^{\alpha+1}}$ & $*$ & $\frac{1}{1+\alpha}$ & $x^{M}\left(\frac{\left(x^{M}\right)^{2}}{\left(x-x^{M}\right)^{2}}-1\right)$ & $-\frac{1}{\alpha+1}$ \\
\hline $\begin{array}{l}\text { Inverse chi-squared } \\
(v>2)\end{array}$ & {$[0 ; \infty)$} & $\frac{x^{-(v / 2)-1}}{2^{v / 2} \Gamma(v / 2)} \exp \left(-\frac{1}{2 x}\right)$ & $*$ & $\frac{1}{2+v}$ & $2 x^{M}\left(\frac{\left(x^{M}\right)^{2}}{\left(x-x^{M}\right)^{2}}-1\right)$ & $-\frac{2}{v+2}$ \\
\hline Beta prime $(\alpha, \beta>1)$ & {$[0 ; \infty)$} & $\propto x^{\alpha-1}(1+x)^{-\alpha-\beta}$ & $*$ & $\frac{\alpha-1}{\beta+1}$ & $\frac{1}{\beta+1}\left(\frac{x^{M}\left(x^{M}+1\right)}{\left(x-x^{M}\right)^{2}}-1\right)$ & $-\frac{1}{\beta+1}$ \\
\hline Pearson $\left(b_{2}>-\frac{1}{2}\right)$ & & - see the & ix - & & $b_{2}-\frac{\chi\left(x^{M}\right)}{\left(x-x^{M}\right)^{2}}$ & $b_{2}$ \\
\hline
\end{tabular}

${ }^{\dagger}$ The symbol $\propto$ indicates that the density function, for fixed parameters, is proportional to the term given in the table; for cumulative distribution functions marked with ${ }^{*}$, there is no closed-form representation. 
Table II. Distributions without strongly $\left(-\frac{1}{2}\right)$-concave density function. ${ }^{\ddagger}$

\begin{tabular}{|c|c|c|c|c|c|}
\hline Name of distribution & $\begin{array}{c}\text { Interval } \\
\quad X\end{array}$ & $\begin{array}{l}\text { P.d.f. } \\
f(x)\end{array}$ & $\begin{array}{l}\text { C.d.f. } \\
F(x)\end{array}$ & $\begin{array}{l}\text { Values } \\
J_{f}^{\prime}\end{array}$ & $\begin{array}{c}\text { Costs } \\
\bar{J}_{f}^{\prime}\end{array}$ \\
\hline Power $(c<1)$ & {$[0 ; 1]$} & $c x^{c-1}$ & $x^{c}$ & $\nexists 0$ & $>0$ \\
\hline Weibull $(c<1)$ & {$[0 ; \infty)$} & $c x^{c-1} \exp \left(-x^{c}\right)$ & $1-\exp \left(-x^{c}\right)$ & $\nexists 0$ & $>0$ \\
\hline Gamma $(c<1)$ & {$[0 ; \infty)$} & $\frac{x^{c-1} \exp (-x)}{\Gamma(c)}$ & * & $\geq 0$ & $>0$ \\
\hline Chi-Squared $(c<2)$ & {$[0 ; \infty)$} & $\frac{x^{(c-2) / 2} \exp (-x / 2)}{2^{c / 2} \Gamma(c / 2)}$ & * & $\geq 0$ & $>0$ \\
\hline Chi $(c<1)$ & {$[0 ; \infty)$} & $\frac{x^{c-1} \exp \left(-x^{2} / 2\right)}{2^{(c-2) / 2} \Gamma(c / 2)}$ & * & $\geq 0$ & $>0$ \\
\hline Beta $(v<1$ or $\omega<1)$ & {$[0 ; 1]$} & $\propto x^{v-1}(1-x)^{\omega-1}$ & * & mixed & mixed \\
\hline Arc-Sine & {$[0 ; 1]$} & $\frac{1}{\pi \sqrt{x(1-x)}}$ & $\frac{2}{\pi} \arcsin (x)$ & $\geq 0$ & $\geq 0$ \\
\hline Pareto $(\beta<1)$ & {$[1 ; \infty)$} & $\beta x^{-\beta-1}$ & $1-x^{-\beta}$ & $<0$ & $>0$ \\
\hline Log-normal $\left(\sigma_{L}^{2}>2\right)$ & {$[0 ; \infty)$} & $\propto \frac{1}{x} \exp \left(-\frac{\left(\ln x-\mu_{L}\right)^{2}}{2 \sigma_{L}^{2}}\right)$ & * & $\begin{array}{l}\text { mixed } \\
\text { (num.) }\end{array}$ & $>0$ \\
\hline Student's $t(n<1)$ & $\mathbb{R}$ & $\propto\left(1+\frac{x^{2}}{n}\right)^{-\frac{n+1}{2}}$ & $*$ & $\begin{array}{l}\geq 0 \\
\text { (num.) }\end{array}$ & $\begin{array}{l}\geq 0 \\
\text { (num.) }\end{array}$ \\
\hline F distribution $\left(m_{1}<2\right.$ or $\left.m_{2}<2\right)$ & {$[0 ; \infty)$} & $\propto \frac{x^{\frac{m_{1}}{2}-1}}{\left(m_{1} x+m_{2}\right)^{\frac{m_{1}+m_{2}}{2}}}$ & * & $\begin{array}{l}\text { mixed } \\
\text { (num.) }\end{array}$ & $>0$ \\
\hline Mirror-image of Pareto $(\beta<1)$ & $(-\infty ;-1]$ & $\beta(-x)^{-\beta-1}$ & $(-x)^{-\beta}$ & $>0$ & $<0$ \\
\hline Log-logistic $(\beta<1)$ & {$[0 ; \infty)$} & $\frac{\beta x^{\beta-1}}{\left(1+x^{\beta}\right)^{2}}$ & $\frac{x^{\beta}}{1+x^{\beta}}$ & $<0$ & $>0$ \\
\hline Inverse gamma $(\alpha<1)$ & {$[0 ; \infty)$} & $\frac{\exp (-1 / x)}{\Gamma(\alpha) x^{\alpha+1}}$ & * & $\begin{array}{c}\geq 0 \\
\text { (num.) }\end{array}$ & $>0$ \\
\hline Inverse chi-squared $(v<2)$ & {$[0 ; \infty)$} & $\frac{x^{-(v / 2)-1}}{\Gamma(v / 2)} \exp \left(-\frac{1}{2 x}\right)$ & $*$ & $\begin{array}{l}\geq 0 \\
\text { (num.) }\end{array}$ & $>0$ \\
\hline Beta prime $(\alpha, \beta<1)$ & {$[0 ; \infty)$} & $\propto x^{\alpha-1}(1+x)^{-\alpha-\beta}$ & $*$ & mixed (num.) & $>0$ \\
\hline Pearson $\left(b_{2}<-\frac{1}{2}\right)$ & & - see the Appen & & mixed (num.) & ixed (num \\
\hline
\end{tabular}

₹ The symbol $\propto$ indicates that the density function, for fixed parameters, is proportional to the term given in the table; for cumulative distribution functions marked with *, there is no closed-form representation; the abbreviation "num." indicates that the result has been obtained through a numerical analysis. 